\title{
VARIOUS SYSTEMS OF SET THEORY BASED ON COMBINATORY LOGIC
}

\author{
M. W. BUNDER
}

1. Introduction We consider in this paper various systems of set theory which, in their usual formulation, are based on predicate calculus. Here the predicate calculus is an applied predicate calculus, in that alongside the various categories of variables we have constants of various categories; concerning these constants we assume certain additional axioms.

In order to translate this situation into combinatory logic the axioms we assume must be such as to make each of these new constants satisfy the proper grammatical condition (see [2]). If that is the case, such constants can be substituted for the variables with the same grammatical conditions, and these grammatical conditions can be detached from the set of premises of such formulas. Sometimes, these constants can be defined in such a way that the only essentially new axiom is that giving the grammatical condition.

One of these constants is the ob A, this is the range of obs over which an ob must be a propositional function before we can apply the deduction theorem (see [3]); we shall also take it as our range of quantification. Thus in this chapter we apply the theorems of [2] with A for $a$. The condition La can be dropped in these theorems as we have $\vdash$ LA by Axiom 8 of [2].

Next we consider the relation $\varepsilon$ which is usually taken as primitive in set theory. In our system $\varepsilon$ can be defined by $\mathrm{Cl}$ so that $x \varepsilon y$ is translated as $y x$. The ob $\mathrm{Cl}$ can be proved to be a predicate as we have in each system an axiom which gives us

$$
\mathbf{A} u, \mathbf{A} v \vdash \mathbf{H}(u v) .
$$

This rule, as was shown in [4], is inconsistent with $E$ for $A$, but with a suitably restricted $A$ there should be no problem. The rule gives us $\mathrm{A} u, \mathbf{A} v \vdash \mathrm{H}(\mathrm{Cl} v u)$ which by the deduction theorem gives us,

$$
\vdash \mathrm{F}_{2} \mathrm{AAH}(\mathrm{Cl}) \text {. }
$$

Other constants that we shall be using are the first order predicates $M$ and $M_{1}$, where $M$ is a new primitive for the category of all sets, and $M_{1}$ is 
the category of all classes. In both cases we will need to have the grammatical condition $\mathrm{FAH} X$ holding, thus we have to have as an axiom: ${ }^{1}$

Axiom M. $-F A H M$.

We do not need to state $\vdash F A H M_{1}$ as an axiom as in the ZermeloFraenkel system we do not have classes and in the other systems we can define $M_{1}$ so that this property holds.

Bernays in his system [1], where he has both sets and classes, also has two sets of variables, one ranging over sets and one over classes. This is not really necessary as it has been proved that these can be reduced to a single set. In our system we can duplicate Bernay's method of having two sets of variables by considering both $M_{1}$ and $M$ as subclasses of FAH. The grammatical conditions then hold for sets and classes and for example the theorem "if $x$ and $y$ are classes then their intersection is a class" becomes:

$$
\mathrm{M}_{1} x, \mathrm{M}_{1} y \vdash \mathrm{M}_{1}(\cap x y) .
$$

Alternatively we could work without such variables and translate the theorem as

$$
\vdash \Xi \mathbf{A}[x]: . \Xi \mathbf{A}[y]: \mathbf{M}_{1} x \supset . M_{1} y \supset M_{1}(\cap x y) .
$$

Any of the systems that we consider, in as far as they do not already have them, can be extended to include individuals. There are three ways of handling this problem. First there is Quine's method where an individual is its own unit set; this requires a modification of the axiom of grounding. Second, we could allow $X Y$ where $X$ is an individual, always to be a (false) proposition, but this requires a modification of the extensionality axiom. A third solution is not to allow $\vdash \mathrm{H}(X Y)$ where $X$ is an individual to be proved in the system. Individuals will in this case not be sets or classes, but they will have to be included in our range of quantification A. Modifications are then needed in axioms in which we quantify over sets or classes. We will not consider these modifications for bringing in individuals any further here.

We define the translation of a set theoretical statement and its grammatical conditions exactly as the translation of a predicate calculus statement in [2]. For the predicate constants $M$ and $M_{1}$ we already have grammatical conditions, we also need them for equality. Set and class equality we define in terms of extensionality, rather than identify them with Q. This is because certain problems arise in connection with the extensionality axiom. We can state this axiom as follows.

$$
\vdash \Xi \Delta[x]: \Xi \Delta[y] .(\Xi \mathbf{A}[u] . x u \sim y u) \supset \mathbf{Q} x y,
$$

1. If we assume $\mathbf{H}=\mathbf{B L K}$ as in [2] we also need to have $\boldsymbol{L} \mathbf{L M}$. If we define $\mathbf{L}$ by FAH this property holds trivially. 
where in the Zermelo-Fraenkel [7] and Bernays systems $\Delta$ is $M$ and in the Gödel system [8] $\mathrm{M}_{1}{ }^{2}$ As a result of such an axiom, theorems such as the Church-Rosser theorem may fail to hold. To avoid this, or at least to keep the possibility of another interpretation open, we shall define set (or class) equality using the extensionality property and then state an axiom to give the other property of equality. Other solutions, such as defining equality by the Leibnitz property, are less satisfactory than the above; statements such as $\vdash L L$ are then required as axioms. We could also of course leave set equality undefined and have two axioms.

2. The Zermelo-Fraenkel System We now consider the Zermelo system of set theory as modified by Fraenkel [7]. The modification means, for one thing, that the class of objects we quantify over in the theory is the class of sets itself, rather than an unspecified class. Thus in the work below we identify $A$ with $M$. One further thing we must specify is that every element $X$ of $\mathrm{M}$ must be such that $\mathrm{H}(X U)$ whenever $U$ is a set. In other words the grammatical condition $\vdash \mathrm{FMH} X$ must hold for all elements $X$ of $\mathrm{M}$.

Axiom MH. $\vdash \Xi M(F M H)$.

Now we consider the Zermelo-Fraenkel axioms. The first of these is extensionality, which we mentioned in the introduction. The definition of set equality which we use to replace it in this system is as follows. ${ }^{3}$

Definition $\mathbf{Q}_{1} . \mathbf{Q}_{1} \equiv[x, y] \Lambda(\mathbf{M} x)(\Lambda(\mathbf{M} y)(\Xi \mathbf{M}[z] x z \sim y z))$.

The required axiom is then:

Axiom $\mathrm{Z}_{1}^{\prime}: \vdash \mathrm{FMH} z \supset_{z}: \Xi \mathrm{M}[x] . \Xi \mathrm{M}[y]\left(\mathbf{Q}_{1} x y \supset . z x \sim z y\right)$.

Using this definition of equality we now show that we can derive a grammatical condition for $Q_{1}$.

Theorem 1. $\vdash \mathrm{F}_{2} \mathrm{MMHQ}_{1}$.

Proof. We have

$$
\begin{aligned}
& \mathbf{M} x \vdash \mathbf{H}(\mathbf{M} x), \\
& \mathbf{M} y \vdash \mathbf{H}(\mathbf{M} y) .
\end{aligned}
$$

Now by Axiom $\mathrm{MH}, \mathrm{M} z, \mathrm{M} x \vdash \mathrm{H}(x z)$, and so $\mathrm{M} x, \mathrm{M} y, \mathrm{M} z \vdash \mathrm{H}(x z \sim y z)$. Thus by Theorem 4 of [2] and $\vdash$ LM,

$$
\mathrm{M} x, \mathrm{M} y \vdash \mathrm{H}(\Xi \mathrm{M}[z] . x z \sim y z) ;
$$

so using (1), (2), and (3) and Definition $Q_{1}$ we get $M x, M y \vdash H\left(Q_{1} x y\right)$, and by $\vdash L M$ and the deduction theorem, $\vdash \mathrm{F}_{2} M M \mathrm{MQ}_{1}$.

The second axiom asserts the existence of a set containing two given objects, this concept can be defined in the system and then specified to be a

2. $\sim$ is used for the equivalence connective.

3. We can prove $\mathbf{M} x, \mathbf{M} y, \mathbf{0} x y \vdash \mathbf{0}_{1} x y$. 
set by means of an appropriate axiom. For this set containing obs $x$ and $y$, we take $\mathbf{U}(\mathbf{Q} x)(\mathbf{Q} y)$, where $\mathbf{U}$ is defined as follows.

Definition $U$. $U \equiv \Phi V$.

Thus $\mathrm{U} x y z=\mathrm{V}(x z)(y z)$, and $\mathrm{U} x y$ can be considered as the union of sets $x$ and $y$. Similarly $\cap$ can be defined as $\Phi \Lambda$. Now the axiom can be stated.

Axiom Z2. $\vdash \mathrm{F}_{2} \mathrm{MMM}\left(\psi \cup \mathrm{Q}_{1}\right)$.

The third axiom states "If the condition $P a$ is definite for each element $a$ of a set $x$ then there exists a set which contains just those elements $a$ of $x$ for which $P a$ holds." Fraenkel takes "is definite" to refer to $P$ alone; this we could represent as $\mathrm{FMH} P$, since then for any set $x$ we have $H(P x)$. The ob which contains those elements $u$ of $x$ such that $y u$ holds will obviously be $\cap y x$, all that has to be done therefore is to specify that $\cap y x$ must be a set under these conditions.

Axiom Z3. $\vdash \mathrm{FMH} y \supset_{y} . \mathrm{FMM}(\cap y)$.

Here if $\mathrm{M} x$ restricts $x$ to be a set, and $\mathrm{FMH} y$ requires $y u$ to be definite (i.e., a proposition) whenever $u$ is an element of $M$, then $M(\cap x y)$ says that the intersection of $x$ and $y$ is a set.

The next axiom is the power set axiom. Cogan in his thesis [5] gives $C \equiv X$ as the class of all subsets of $X$ and goes on to state an axiom to make any such class a set. He also has an axiom to insure that the intersection of any set and any class is a set, so a subset of a set must be a set. Here we can do without the second axiom by taking $\cap M(C \Xi X)$ as the set of all subsets of a set $X$. This leaves open the questions as to whether, for some sets $X$, there may exist objects $Y$ which are not sets, (i.e., individuals), such that $\Xi Y X$. Such a $Y$ will not necessarily satisfy the condition of Axiom Z3. Axiom Z4 therefore becomes:

Axiom Z4. $-\mathrm{FMM}([x] . \cap \mathrm{M}(\mathrm{C} \Xi x))$.

Note that this can also be written as $\vdash \operatorname{FMM}\{P w\}$ where $\{P w$ ' relation" defined by $[x, y] . \Lambda(M y)(\Xi y x)$.

With Axioms Z4 and Z2 we can prove the two other parts of Zermelo's original Axiom 2. ${ }^{4}$ These asserted the existence of an empty set and a set containing a given element.

Theorem 2. (a) There exists an empty set. (b) For a given set there exists a set containing the given set as sole element.

Proof. (a) By Theorem 1 we have $M x \vdash H\left(W Q_{1} x\right), M x \vdash H\left(B-\left(W Q_{1}\right) x\right)$, and so $\vdash F M H\left(B-\left(W Q_{1}\right)\right)$. Now by Axiom Z3 $M x \vdash M\left(\cap\left(B-\left(W Q_{1}\right)\right) x\right)$. The later axioms such as the axiom of infinity assert the existence of a set, thus substituting any set $X$ for $x$ gives $\vdash M\left(\cap\left(B-\left(W Q_{1}\right)\right) X\right)$. This will be unique by extensionality.

4. This is similar to the proof in Fraenkel and Bar Hillel. For Zermelo's original axioms see [10]. 
(b) If the set $x$ is the empty set 0 we have by Axiom $Z 4, \vdash M(\cap M(C \Xi 0))$. If we have the extra axiom:

Axiom 12. $\vdash \Lambda x y \supset_{x, y} x$

we can say $\cap M(C \Xi 0) x \vdash Q_{1} 0 x$ so that $\cap M(C \Xi 0)$ contains only the given set 0 .

If $x$ is nonempty then by (a) and Axiom 2, $\mathrm{U}\left(\mathrm{Q}_{1} x\right)\left(\mathrm{Q}_{1} 0\right)$ is a set and Axiom 3 gives us that $\cap\left(U\left(Q_{1} x\right)\left(Q_{1} 0\right)\right)\left(Q_{1} x\right)$ is a set. This of course contains only $x$.

Note that we cannot prove that $B-\left(W Q_{1}\right)$ and $Q_{1} x$ are sets given that $x$ is one, as we can only apply extensionality to prove these equal to the above sets if they are already sets. We could simply take $\vdash M\left(B-\left(W Q_{1}\right)\right)$ and $-F M M Q_{1}$ as axioms (and grammatical conditions); we would then not need to use Axiom 12.

Axiom $\mathrm{Z} 5$ is the sum set axiom. Given a set $Y$ the class of all elements of elements of $Y$ can be given by $\left\{U_{n}\right\} Y$, where,

Definition $\left\{U_{n}\right\} . \quad\left\{U_{n}\right\} \equiv[y, x] \Sigma M[u](\Lambda(u x)(y u))$.

For this to be a set is needed:

Axiom Z5. -FMM\{Un\}.

The sixth axiom is the axiom of choice. Zermelo's version of it can be translated into the combinatory system, but its meaning will be clearer if we use a notation which is closer to the classical one. Such a notation will now be defined.

Definition $\wedge . x \wedge y \equiv \Lambda x y$.

Definition $\vee . x \vee y \equiv \mathrm{V} x y$.

We will not introduce $(\forall x)$ for $\Xi M[x]$ and $(\exists x)$ for $\Sigma M[x]$, as we may not always want to identify $A$ with $M$. We now state the axiom of choice.

Axiom Z6. $\vdash \Xi \mathbf{M}[x]: .(\Xi \mathbf{M}[u]: \Xi \mathbf{M}[v] . x u \wedge x v \wedge u \neq v \supset \cap u v=0 \wedge u \neq 0): \supset:$ $\Sigma \mathbf{M}[t] \Xi \mathbf{M}[w] . t w \supset\{\mathbf{U n}\} x w \wedge\left(\Xi \mathbf{M}[u]: x u . \supset . \Sigma \mathbf{M}[s] \cap t u=\mathbf{Q}_{1} s\right)$.

Thus if $X$ is a set, the elements of which are nonempty disjoint sets, then their union $\left\{U_{n}\right\} X$ contains at least one subset $T$ which has one and only one element in common with every element of $X$.

The remaining axiom is the axiom of infinity. This can be treated as Cogan [5] does, by taking a new primitive $\{\operatorname{lnf}\}$ with the following axioms:

Axiom Z7. (a) $\vdash\{\operatorname{lnf}\} 0$.

(b) $\vdash M\{\operatorname{lnf}\}$.

(c) $\vdash \Xi M[x] .\{\operatorname{Inf}\} x \supset\{\operatorname{lnf}\}\left(Q_{1} x\right)$.

As an alternative to (c) we could take the von Neumann form: $1 \Xi M[x]$. $\{\operatorname{lnf}\} x \supset\{\operatorname{lnf}\}(\mathrm{U} x(\mathbf{Q} x))$.

The sets $\{\operatorname{lnf}\}$ will be infinite in the usual sense as it will not be possible for an element $\mathbf{Q}_{1}\left(\mathbf{Q}_{1} \ldots\left(\mathbf{Q}_{1} 0\right) \ldots\right)$ with $k \mathbf{Q}_{1}^{\prime}$ s to be equal to $Q_{1}\left(Q_{1} \ldots\left(Q_{1} 0\right) \ldots\right)$ with $l Q_{1}^{\prime} \mathbf{S}$. If it were possible then by rules $(\mu)$ and 
Eq $q^{5}$ we would get: $\mathbf{Q}_{1}\left(\mathbf{Q}_{1} \ldots\left(\mathbf{Q}_{1} 0\right) \ldots\right)=0$, where there are $k-l \mathbf{Q}_{1}^{\prime} \mathbf{S}$ and this is impossible, as it leads to $\vdash 0\left(Q_{1} \ldots\left(Q_{1} 0\right) \ldots\right)$ with $k-l-1 Q_{1}^{\prime}$.

It is also possible to define $\{\mathrm{Clnf}\}$ as follows:

Definition $\{\mathrm{CInf}\} .\{\mathrm{CInf}\} \equiv[x]: \mathrm{M} x . \wedge . x 0 . \wedge \Xi \mathrm{M}[u] . x u \supset x\left(\mathrm{Q}_{1} u\right)$,

the axiom of infinity then asserts the existance of such a set, i.e.,

Axiom $\mathrm{Z7}^{\prime}$. $1-\Sigma \mathrm{M}\{\mathrm{Clnf}\}$.

The above definition of $\{C \ln \}$ is closest to what Zermelo expressed in Axiom Z7 (c), but an alternative with some advantages is von Neumann's definition, with which we can use the same Axiom Z7'.

Definition $\{\mathrm{CInf}\}(2) .\{\mathrm{C} \operatorname{lnf}\} \equiv[x]: \mathbf{M} x . \wedge . x 0 . \wedge \Xi \mathrm{M}[u] . x u \supset x\left(\mathrm{U} u\left(\mathrm{Q}_{1} u\right)\right)$.

The Zermelo system is extended to the Zermelo-Fraenkel system by adding two further axioms, namely the axioms of grounding and replacement. The first of these states that every nonempty set has an element that has no elements in common with it. This can be represented as follows.

Axiom Z8. $\vdash \Xi M[x]: x \neq_{1} 0 . \supset . \Sigma M[y] . x y \wedge\left(\cap x y={ }_{1} 0\right){ }^{6}$

The replacement axiom requires that given a nonempty set $x$ and a single valued function $f$ (i.e., $\vdash$ FMM $f$ ), there is a set $y$ such that $\vdash y u$ if and only if there is a set $v$ contained in $x$ such that $f v={ }_{1} u_{0^{7}}$ An ob $\{\operatorname{Rep}\}$ is defined so that this axiom can be expressed.

Definition $\{\operatorname{Rep}\} . \quad\{\operatorname{Rep}\}=[f, x, u] \Sigma M[v] . \Lambda(x v)\left(f v={ }_{1} u\right)$.

The axiom is then represented by: ${ }^{8}$

Axiom Z9. $-F M M f \supset_{f} . \operatorname{FMM}(\{\operatorname{Rep}\} f)$.

Now we can prove the general theorem for the Zermelo-Fraenkel system.

Theorem 3. If $T$ is a theorem of Zermelo-Fraenkel set theory and $N$ is the set of grammatical conditions for $T^{\prime}$, the translation of $T$ into the combinatory system, then $N \vdash T^{\prime}$.

Proof. This goes exactly as the proof of Theorem 25 of [2] except that there are some extra axioms to consider, namely those of the ZermeloFraenkel set theory itself. It $T$ is the extensionality axiom $\vdash T^{\prime}$ follows from Definition $Q_{1}$. $T^{\prime}$ contains no variables and only the constant predicate $Q_{1}$ for which we have obtained the correct grammatical condition in

5. These are as in [6]

( $\mu$ ) If $\mathbf{0}_{1} X Y$ then $\mathbf{0}_{1}(Z X)(Z Y)$

Eq. $\mathbf{a}_{1} X Y, X \vdash Y$.

6. $z={ }_{1} w$ stands for $\mathbf{O}_{1} z w$.

7. In the case of an empty set the replacement set is trivially empty.

8. Axiom Z3 becomes provable when Z9 is added (See Fraenkel and Bar Hillel [7]). 
Theorem 1. The other property of equality $\left(\mathrm{Z}_{1}^{\prime}\right)$ is in the correct translated form if we apply Rule $\Xi$ once. Axioms $\mathrm{Z} 2, \mathrm{Z} 4$ and $\mathrm{Z} 5$ are themselves grammatical conditions for certain constants, similarly Axioms Z3 and Z9 become schemes for grammatical conditions if we apply Rule $\Xi$ with argument $z$. Axioms $\mathrm{Z} 6, \mathrm{Z} 7$ and $\mathrm{Z} 8$ are thus the only axioms not giving grammatical conditions, these assert the existence of certain sets.

Axiom Z6 contains no free variables and the only constant in it we have, as yet, no grammatical condition for is $\cap$, however $\vdash F_{2} M M M \cap$ follows by Axioms $\mathrm{Z3}$ and $\mathrm{MH}$. In Axiom $\mathrm{Z} 7$ we have the new primitive predicate $\{$ Inf $\}$, but by Axioms $\mathrm{Z7}$ (b) and $\mathrm{MH}$ this has the correct grammatical condition $\vdash$ FMH $\{$ Inf $\}$. Similarly we can easily prove $\vdash F M H\{C \operatorname{lnf}\}$ if we use Axiom Z7'. Axiom Z8 also contains no variables and as constants only $0, \cap$ and $Q_{1}$ for which we have the grammatical conditions. Thus all of the axioms have the correct form and the proof of the theorem goes through as for Theorem 25.

3. Bernays' System Now Bernays' system [1] will be considered. It contains not only sets, as Zermelo's system does, but also classes. The basic idea of a class is that one is determined by every predicate over sets. This property is expressed formally by Church's scheme, as given by Bernays, which, in classical notation is given by:

$$
x \varepsilon\{x \mid \phi(x)\} \sim \phi(x)
$$

whenever $x$ is a set.

As Bernays only has sets as objects, talks of predicate over sets and quantifies only over sets, we can again let $A$ be $M$ in this section. $A$ "predicate over sets" will thus be an ob $X$ such that $\vdash \mathrm{FMH} X$, and such that $\vdash L X$. By the use of this a simple combinatory logic version of Church's scheme can be proved. In combinatory logic the concepts " $x$ is an element of $y$ " (or $x \varepsilon y$ ) and " $x$ has the property $y$ "' (or $y(x)$ ) can both be expressed by $y x$. Instead of Church's scheme we then get:

$$
\mathrm{FMH} y, \mathbf{M} x+y x \sim y x .
$$

(Note that FMH $y$ and $\mathrm{M} x$ are the grammatical conditions for $y x \sim y x$.)

A class in the Bernays sense (the category of these we will denote by $M_{1}$ ) must therefore have the property $\mathrm{FMH}$. At this point however we have some further alternatives. We can leave $M_{1}$ as primitive and have $\vdash M_{1} x \supset_{x} F M H x$, as an axiom; we can define $M_{1}$ as $F M H$, which will be an extension of Bernays' system as we will have $1 F M H(W Q)$ and thus a class which will contain obs other than sets; or we can define $M_{1}$ by $\cap(F M H)(C \equiv M)$, thus restricting classes to obs containing only sets. Here we shall use the second of these approaches. Bernays has no axioms to say that all elements of classes must be sets and FMH also has the advantage of being a grammatical condition.

We now define class equality as we did set equality in the previous section.

Definition $Q_{1}^{\prime} . Q_{1}^{\prime} \equiv[x, y]\left(\Xi M[u] . x u \sim y u . \wedge . M_{1} x . \wedge . M_{1} y\right)$. 
Especially if we assume $M_{1}$ to be $F M H$ it is better not to assume that $Q_{1}^{\prime}$ is the same as $Q$. In that case it is conceivable that there might be two classes which have all their sets in common, but which are not equal combinatorially. With $Q_{1}^{\prime}$ as it is however, we can prove

$$
\mathbf{M}_{1} x, \mathbf{M}_{1} y, x={ }_{1} y \vdash \Xi \mathbf{A}[u] . x u \sim y u,
$$

using our version of Church's scheme.

Next Bernays has two axiom schemes for equality over sets.

E1. $\mathrm{M}_{1} z, \mathrm{M} x, \mathrm{M} y \vdash x={ }_{1} y . \supset . z x \supset z y$.

E2. $\mathrm{M} x, \mathrm{M} y \vdash \Xi \mathrm{M}([z] . x z \sim y z)$. ว. $x={ }_{1} y$.

In his system he has a certain class "represented" by a set if it has the same sets as elements as that set. In our system where we can compare sets and classes more easily the "representation" can be replaced by something similar to the notion $Q_{1}$.

Definition $\mathbf{Q}_{0} . \mathbf{Q}_{0} \equiv[x, y] . \mathbf{M}_{1} x \wedge \mathbf{M} y \wedge \Xi \mathbf{M}[u] . x u \sim y u$.

If we define set equality by $Q_{1}$ as in the previous section we have E2 as a result of the definition and the following assumption.

Axiom $M_{1}$ M. $\vdash \Xi M(F M H)$,

which is equivalent to the grammatical condition $\vdash \mathbf{F}_{2} \mathbf{M M H I}$.

We then can derive $\mathrm{E} 1$ from a generalisation of Axiom $Z_{1}^{\prime}$, viz.

Axiom $\mathrm{E} 1^{\prime} . \vdash \mathrm{M}_{1} z \supset_{z} \Xi \mathrm{M}[x] \Xi \mathrm{M}[y]: x={ }_{1} y \supset . z x \supset z y$.

Of the remaining axioms $A 1$ asserts the existence of an empty set. This we can represent as:

Axiom BA1. $-\mathrm{M}\left(\mathrm{B}-\left(\mathrm{WQ}_{1}\right)\right.$.

Note that we write the combinatory versions of Berneys' $\mathrm{A} 1, \ldots$ as BA1, . . . His second says that a set with an extra element added is a set. This can be stated in the following way.

Axiom BA2. $-F_{2} M M M\left(C(B B U) Q_{1}\right)$.

(This could be replaced by $\vdash \mathrm{F}_{2} \mathrm{MMMU}$ and $\vdash F M M Q_{1}$.)

Axiom A3 asserts that if $z$ is a function over sets (i.e., an element of FMM) then the union of all the sets $z x$ where $\vdash y x$ and $\vdash M y$, is again a set. This can be represented by $\{R p\}$ which is defined by,

Definition $\{\operatorname{Rp}\} . \quad\{\mathbf{R p}\} \equiv[z, y, u] . \Sigma \mathbf{M} .[x] \wedge(y x)(z x u)$.

All that is needed then is an axiom to assert that $\{\mathbf{R} p\} z y$ is a set, under the required conditions.

Axiom BA3. $\vdash \mathrm{F}_{2}(\mathrm{FMM}) \mathrm{MM}\{\mathrm{Rp}\}$.

Thus $\mathrm{FMM} z, \mathbf{M} y \vdash \mathbf{M}(\{\mathbf{R p}\} z y)$. Axioms BA2 and BA3 in this system replace Axioms Z2 and Z3 of Zermelo's system. Axioms A4, A5 and A7 are the power set axiom, the axiom of choice and the axiom of grounding and for these $\mathrm{Z} 4, \mathrm{Z} 6$ and $\mathrm{Z} 8$ can be used. 
Axiom A6 is Bernays' axiom of infinity. He defines natural numbers, in terms of a zero ordinal and its successors and then has an axiom to the effect that the class $\mathrm{Nn}$ of these is a set. Instead of this we can have Definition $\{$ Clnf $\}$ and Axiom Z7'.

Thus we have obtained all the axioms of Bernays' system and we can prove the general theorem for the Bernays' system.

Theorem 4. If $T$ is a theorem of Bernays' set theory and $N$ is the set of grammatical conditions for $T^{\prime}$, the translation of $T$ into the combinatory system, then $N \vdash T^{\prime}$.

Proof. This again is proved as Theorem 25 of [2]. We have a theorem with the correct grammatical conditions to correspond to Church's scheme. Also we have Axiom E2 proved from Definition $Q_{1}$. Axiom schemes corresponding to Bernays' Axiom Schemes E1 and A3 follow by one use of Rule $E$ and our Axioms $E 1$ ' and BA3, also with the correct grammatical conditions. Axiom BA2, itself a grammatical condition, is the translation of Bernays' A2. The remaining axioms are handled as they were in the Zermelo-Fraenkel system. Thus we have all the axioms of Bernays in the correct form and the theorem is proved.

4. Gödel's Set Theory As in the other two systems of set theory we shall base the system on the predicate calculus we developed in section 3, taking this time $A$ as the set of all objects. This $A$ cannot be $M$ as it must include $M_{1}$; but it is possible to replace $A$ by $M_{1}$, the category of all classes, as in the system $M$ is a subclass of $M_{1}$. Below we will use $A$ keeping this possibility in mind, and also leaving open the possibility of $A$ containing individuals and perhaps relations, as well as classes. It is certain in any case that we will need the following as an axiom.

Axiom $M_{1}$. $\vdash \Xi M_{1} A$.

Gödel's notion of a class is somewhat more restricted than that of Bernays in that he has an axiom to the effect that all members of classes must be sets. For Bernays $X \varepsilon Y$ where both $X$ and $Y$ are classes is not defined, however in the previous section we extended his system to include this possibility. Also Gödel has an axiom to ensure that all sets are also classes, whereas Bernays has certain classes represented by sets, if they have the same elements. The two axioms of the Gödel system that were mentioned above, can be written as follows. ${ }^{9}$

Axiom GA1. $\vdash \Xi M[x] . M x \supset M_{1} x$.

Axiom GA2. $\vdash \Xi \mathbf{A}[x]: \mathbf{M}_{1} x \supset: \Xi \mathbf{A}[y] . x y \supset \mathbf{M} y$.

Gödel's Axiom A3 is identical to Bernays' axiom of extensionality for

9. Cogan [5] had as a definition $\mathbf{M}_{1}=\mathbf{F M H}$. This gives us obs such as K(WOS) as classes, which have elements which are not necessarily sets. This is Titgemeyer's paradox. This section gives a solution to this paradox. See [9]. 
classes, for class equality we can use the same definition, with $\mathbf{A}$ for $\mathbf{M}$. Also we need Axiom $\mathrm{Z}_{1}^{\prime}$ with $\Xi A[x] \cdot \mathrm{M}_{1} x \supset^{\prime \prime}$ instead of $\mathrm{M}_{1} x \supset_{x}^{\prime \prime}$, and $\mathrm{Q}_{1}^{\prime}$ as the equality.

The fourth axiom of the A group is the pair set axiom which we can state as follows.

Axiom GA4. $\vdash \Xi \mathbf{A}[x] . \Xi \mathbf{A}[y] . \mathbf{M} x \supset . \mathbf{M} y \supset \mathbf{M}\left(\mathbf{U}\left(\mathbf{Q}_{1}^{\prime} x\right)\left(\mathbf{Q}_{1}^{\prime} y\right)\right)$.

Now we consider the $\mathrm{C}, \mathrm{D}$ and $\mathrm{E}$ axioms. There are four axioms in group $\mathrm{C}$, these are the axiom of infinity, the sum set axiom, the power set axiom and the "definiteness axiom"; they can all be formulated as in the first section with $\Xi \mathbf{A}[u] \mathbf{M} u \supset$ and $\Sigma \mathbf{A}[u] \mathbf{M} u \wedge$ instead of $\Xi \mathbf{A}[u]$ and $\Sigma \mathbf{A}[u]$. This can also be done for Axioms $\mathrm{D}$ and $\mathrm{E}$, the axioms of grounding and choice.

Note that in the other two systems some of the axioms were themselves grammatical conditions for some constant ob. With our replacement of quantification over $M$ by quantification over $A$ statements such as -FMM $\{$ Un $\}$ no longer constitute grammatical conditions, and to fit in the predicate calculus this has to be replaced by $\vdash \Xi \mathbf{A}[u] . \mathbf{M} u \supset \mathbf{M}\{$ Un $\} u)$. Also we need a grammatical condition for $\left\{U_{n}\right\}$, viz.

Axiom A $\{U n\}$. $\vdash$ FAA $\{U n\}$.

Similarly we need:

Axiom $A \cap . \vdash F_{2} A A A \cap$.

Axiom A\{Inf $\}$. - FAA $\{\operatorname{lnf}\}$.

Axiom Asubc. $\operatorname{FAA}([y] . \cap \mathrm{M}(\mathrm{C} \Xi y))$.

Thus we have the $\mathrm{C}, \mathrm{D}$ and $\mathrm{E}$ axioms in their correct translated form with the appropriate grammatical conditions. The $\mathrm{A}$ axioms are also in the correct form if we have:

Axiom AU. $-F_{2}$ AAAU.

Axiom $A Q_{1}$. $\vdash F A Q_{1}^{\prime}$.

Axiom $H Q_{1}$. $\vdash F_{2} A A H Q_{1}^{\prime}$.

The last of these will be derivable from Definition $Q_{1}$ and Axiom HA which we introduce later on. In addition to these we need $\vdash \Xi M_{1}(F A H)$ and $\vdash F A H M_{1}$ but these become derivable when we define $M_{1}$ below.

Gödel's set of axioms B deals with relations and classes, using this set of axioms he later proves his "general existence theorem" from which his axioms are then derivable. In our system it seems more natural to define relations as many-placed predicates rather than as classes or ordered $n$-tuples of sets. We shall therefore prove Gödel's general existence theorem directly using definitions of classes and relations and then show that this method is equivalent to Gödel's.

Definition $\mathbf{M}_{n} . \mathbf{M}_{n} \equiv[x] . \mathbf{F}_{n} \mathbf{A} \ldots \mathbf{A H} x . \wedge . \Xi \mathbf{A}\left[x_{1}\right] \ldots \Xi \mathbf{A}\left[x_{n}\right] . x x_{1} \ldots x_{n} \supset$ $\mathrm{M} x_{1} \wedge \ldots \wedge \mathrm{M} x_{n}$.

In particular $\mathbf{M}_{1} \equiv[x] . \mathrm{FAH} x \wedge . \Xi \mathbf{A}\left[x_{1}\right] . x x_{1} \supset \mathbf{M} x_{1}$. This definition of $\mathbf{M}_{1}$ we 
will take for Gödel's primitive notion, the definitions of relations can be regarded as temporary and as a means by which we indicate how Gödel's work could be carried out without the use of ordered pairs. Gödel's definition of relations can then be used as he does, in terms of $M$ and $M_{1}$. Note that Axiom GA2 is now derivable.

Before proving the theorem we require a number of assumptions. First we need Axiom $12^{10}$ which gives

$$
\begin{aligned}
& \Lambda x y \vdash x, \\
& \Lambda x y \vdash y,
\end{aligned}
$$

for which we could take $\vdash \Lambda x y \supset_{x, y} y$ as an extra axiom. Also the universe $A$ has to be restricted in such a way that we have:

Axiom HA. $\vdash \Xi A(F A H)$.

This together with Definition $M_{1}$ gives us $1 F A H M_{1}$. This property would seem most natural if we identified $A$ and $M_{1}$, but then our definition of $M_{1}$ would circular, as $M$ is defined in terms of $A$. We could make the identification and then have what is in Definition $M_{1}$ in the form of two axioms. In the work below we leave this question open.

Before stating his general existence theorem Gödel had an inductive definition; this we shall repeat writing " $x y$ " for " $y \in x$, , " $\Sigma \mathbf{A}[u]$. Mu^" for $(\exists u)$ etc.

Definition ppf. ${ }^{11}$ (i) If $X$ and $Y$ are sets or special classes ${ }^{12}$ then $X Y$ is a $p p f$.

(ii) If $X$ and $Y$ are ppfs, then so are $-X$ and $\Lambda X Y$.

(iii) If $X$ is a ppf then so is $\Sigma \mathbf{A}[u] . \mathrm{M} u \wedge X$, and if provided $\vdash \mathrm{M} x, X$ is a ppf then provided $\vdash \mathrm{M} u,[x] X u$ is a $p p f_{.}{ }^{13}$

Theorem 5. (The general existence theorem for ppfs.) If $\phi\left(x_{1}, \ldots, x_{n}\right)$ is a ppf containing no free variables other than at most $x_{1}, \ldots, x_{n}$; then there exists an ob $X$ such that $\vdash \mathrm{M}_{n} X$ and $\mathrm{M} x_{1}, \ldots, \mathrm{M} x_{n} \vdash X x_{1} \ldots x_{n} \sim \phi\left(x_{1}, \ldots, x_{n}\right)$.

Proof. By induction on the structure of $\phi\left(x_{1}, \ldots, x_{n}\right)$.

Case 1. $\phi$ has no logical operators. Then $\phi\left(x_{1}, \ldots, x_{n}\right)$ is $x_{s} x_{r}$ or $\mathbf{A}_{k} x_{r}$ (where $A_{k}$ is a special class). The case $G_{k}$ (where $G$ is a set or class) can be represented as $\Sigma A[x] . M x \supset \wedge\left(\mathbf{Q}_{1}^{\prime} x \mathbf{A}_{k}\right)(\mathbf{G} x)$ by Axiom GA2, and $\mathbf{Q}_{1}^{\prime} x \mathbf{A}_{k}$ by $\Xi \mathrm{A}[u]: \mathbf{M} u \supset . x u \sim \mathbf{A}_{i} u$ by Axiom GA3. Now let

10. It is possible to avoid the use of this axiom if we introduce a number of extra primitives and further axioms.

11. This notion Gödel calls a "primitive propositional function." It is not a function in our sense; it is one only in the sense that it may involve parameters.

12. These Gödel leaves unspecified.

13. Gödel had' a fourth clause which said that ppf's could only be formed as in clauses (i), (ii) and (iii). This we take to be understood in inductive definitions. In our system, where we can do without variables and our functions do not involve them, the last part of condition (iii) is really irrelevant. 


$$
\mathbf{X}=\left[x_{1}, \ldots, x_{n}\right] \cdot x_{s} x_{r} \wedge \mathrm{M} x_{1} \wedge \ldots \wedge \mathrm{M} x_{n} .
$$

Then by propositional calculus and $\mathrm{M} x_{s} \vdash \mathrm{FMH} x_{s}$, we know $\mathrm{M} x_{1}, \ldots$, $\mathrm{M} x_{n} \vdash \mathrm{X} x_{1} \ldots x_{n} \sim x_{s} x_{r}$. As

$$
\mathbf{A} x_{1}, \ldots, \mathbf{A} x_{n} \vdash \mathbf{H}\left(\mathbf{X} x_{1} \ldots x_{n}\right)
$$

by Axioms $\mathrm{M}$ and $\mathrm{HA}$, we have

$$
\vdash \mathrm{F}_{n} \mathbf{A} \ldots \mathrm{AHX} \text {. }
$$

By (2) we have $\mathrm{X} x_{1} \ldots x_{n} \vdash \mathrm{M} x_{1} \wedge . . \wedge \mathrm{M} x_{n}$; so by (3) $\mathrm{A} x_{1}, \ldots, \mathrm{A} x_{n} \vdash \mathrm{X} x_{1} \ldots$ $x_{n} \supset \mathbf{M} x_{1} \wedge \ldots \wedge \mathbf{M} x_{n}$, and thus $\vdash \Xi \mathbf{A}\left[x_{1}\right] \ldots \Xi \mathbf{A}\left[x_{n}\right] . \mathbf{X} x_{1} \ldots x_{n} \supset \mathbf{M} x_{1} \wedge \ldots$ $\wedge \mathrm{M} x_{n}$. Thus by (4) we have $\vdash \mathrm{M}_{n} X$. Similarly we prove this for

$$
\mathbf{X}=\left[x, \ldots x_{n}\right] \mathbf{A}_{k} x_{r} \wedge \mathbf{M} x_{1} \wedge \ldots \wedge \mathbf{M} x_{n} .
$$

Case 2. Here we assume that $\phi$ has $m$ of the logical connectives,$- \Lambda$ and $(\Sigma \mathbf{A}[u] \mathbf{M} u \wedge)$ and that the theorem holds for $\phi$ 's with fewer logical connectives. $\phi$ can take three forms.

Form (i): $\phi\left(x_{1}, \ldots, x_{n}\right)=-\left(\psi\left(x_{1}, \ldots, x_{n}\right)\right)$ where $\mathbf{M} x_{1}, \ldots, \mathbf{M} x_{n} \vdash \mathbf{Y} x_{1} \ldots x_{n} \sim \psi\left(x_{1}, \ldots, x_{n}\right)$, and $\vdash \mathbf{M}_{n} \mathbf{Y}$. Now let

$$
\mathbf{X}=\left[x_{1}, \ldots, x_{n}\right]-\left(\mathbf{Y} x_{1} \ldots x_{n}\right) \wedge \mathrm{M} x_{1} \wedge \ldots \wedge \mathrm{M} x_{n} .
$$

Then as $\mathbf{A} x_{1}, \ldots, \mathbf{A} x_{n} \vdash \mathbf{H}\left(\mathbf{Y} x_{1} \ldots x_{n}\right)$, we have $\mathbf{A} x_{1}, \ldots, \mathbf{A} x_{n} \vdash \mathbf{H}\left(\mathbf{X} x_{1} \ldots\right.$ $\left.x_{n}\right)$, and thus $1-F_{n} M \ldots$. AHX. The other properties of $\mathbf{X}$ we obtain as in Case 1 , so $\vdash \mathbf{M}_{n} \mathbf{X}$. Also $\mathbf{M} x_{1}, \ldots, \mathbf{M} x_{n} \vdash \mathbf{X} x_{1} \ldots x_{n} \sim-\left(\mathbf{Y} x_{1} \ldots x_{n}\right)$ so $\mathrm{M} x_{1}, \ldots, \mathrm{M} x_{n} \vdash \mathrm{X} x_{1} \ldots x_{n} \sim \phi\left(x_{1}, \ldots, x_{n}\right)$.

Form (ii): $\phi\left(x_{1}, \ldots, x_{n}\right)=\psi\left(x_{1}, \ldots, x_{n}\right) \wedge \chi\left(x_{1}, \ldots, x_{n}\right)$,

where by the inductive hypothesis there is a $\mathbf{Y}$ and a $\mathbf{Z}$ such that, $\mathbf{M} x_{1}, \ldots$, $\mathrm{M} x_{n} \vdash \mathrm{Y} x_{1} \ldots x_{n} \sim \psi\left(x_{1}, \ldots, x_{n}\right), \mathrm{M} x_{1}, \ldots, \mathrm{M} x_{n} \vdash \mathrm{Z} x_{1} \ldots x_{n} \sim \chi\left(x_{1}, \ldots, x_{n}\right)$, and $\vdash \mathbf{M}_{n} \mathbf{Y}$ and $\vdash \mathbf{M}_{n} \mathbf{Z}$. Let

$$
\mathbf{X}=\left[x_{1}, \ldots, x_{n}\right] \mathbf{Y} x_{1} \ldots x_{n} \wedge \mathbf{Z} x_{1} \ldots x_{n} \wedge \mathrm{M} x_{1} \wedge \ldots \wedge \mathbf{M} x_{n} .
$$

Then similarly all the necessary properties hold for $\vdash M_{n} X$.

Form (iii): $\phi\left(x_{1}, \ldots, x_{n}\right)=\Sigma \mathbf{A}[u] \mathbf{M} u \wedge \theta\left(u, x_{1}, \ldots, x_{n}\right)$

where $\mathbf{M} x_{1}, \ldots, \mathbf{M} x_{n}, \mathbf{M} u \vdash \theta\left(u, x_{1}, \ldots, x_{n}\right) \sim \mathrm{T} u x_{1} \ldots x_{n}$ and $\vdash \mathbf{M}_{n+1} \mathbf{T}$. Then let

$$
\mathbf{X}=\left[x_{1}, \ldots, x_{n}\right] \Sigma \mathbf{A}\left([u] \mathbf{M} u \wedge \mathbf{T} u x_{1} \ldots x_{n}\right) \wedge \mathbf{M} x_{1} \wedge \ldots \wedge \mathbf{M} x_{n}
$$

and again all properties hold. Thus the theorem holds in all cases.

Gödel also states a generalisation of this to "normal functions," this proof however does not depend on any of his assumptions concerning relations so it would be trivial to repeat it here.

To prove our method of dealing with relations equivalent to that of Gödel we have to show that for every one of our $n$-ary relations $X$ we have a class $Y$ such that $\mathrm{M} x_{1}, \ldots, \mathrm{M} x_{n} \vdash X x_{1} \ldots x_{n} \sim Y\left\langle x_{1}, \ldots, x_{n}\right\rangle$ where the ordered $n$-tuple $\left\langle x_{1}, \ldots, x_{n}\right\rangle$ is defined as follows. 
Definition $\left\langle x_{1}, \ldots, x_{n}\right\rangle$.

$\left\langle x_{1}, x_{2}\right\rangle \equiv \mathbf{U}\left(\mathbf{Q}_{1}^{\prime}\left(\mathbf{U}\left(\mathbf{Q}_{1}^{\prime} x_{1}\right)\left(\mathbf{Q}_{1}^{\prime} x_{1}\right)\right)\right)\left(\mathbf{Q}_{1}^{\prime}\left(\mathbf{U}\left(\mathbf{Q}_{1}^{\prime} x_{1}\right)\left(\mathbf{Q}_{1}^{\prime} x_{2}\right)\right)\right)$,

$\left\langle x_{1}, \ldots, x_{n}\right\rangle \equiv \mathbf{U}\left(\mathbf{Q}_{1}^{\prime}\left\langle x_{1}, \ldots, x_{n-1}\right\rangle\right)\left(\mathbf{Q}_{1}^{\prime}\left(\mathbf{U}\left(\mathbf{Q}_{1}^{\prime}\left\langle x_{1}, \ldots, x_{n-1}\right\rangle\right)\left(\mathbf{Q}_{1}^{\prime} x_{n}\right)\right)\right)$.

We can prove that there is such a class. First we shall show that any ordered $n$-tuple of sets is a set.

Lemma 1. $\mathrm{M} x_{1}, \ldots, \mathrm{M} x_{n} \vdash \mathrm{M}\left\langle x_{1}, \ldots, x_{n}\right\rangle$.

Proof. From Axioms GA4, GA1 and $\mathbf{M}_{1}$ we get, $\mathbf{M} x_{1} \vdash \mathbf{M}\left(\mathbf{U}\left(\mathbf{Q}_{1}^{\prime} x_{1}\right)\left(\mathbf{Q}_{1}^{\prime} x_{1}\right)\right)$ and also $\mathbf{M} x_{1}, \mathbf{M} x_{2} \vdash \mathbf{M}\left(\mathbf{U}\left(\mathbf{Q}_{1}^{\prime} x_{1}\right)\left(\mathbf{Q}_{1}^{\prime} x_{2}\right)\right)$. Thus again by Axiom GA4,

$$
\mathbf{M} x_{1} \mathbf{M} x_{2} \vdash \mathbf{M}\left(\mathbf{U}\left(\mathbf{Q}_{1}^{\prime}\left(\mathbf{U}\left(\mathbf{Q}_{1}^{\prime} x_{1}\right)\left(\mathbf{Q}_{1}^{\prime} x_{1}\right)\right)\right)\left(\mathbf{Q}_{1}^{\prime}\left(\mathbf{U}\left(\mathbf{Q}_{1}^{\prime} x_{1}\right)\left(\mathbf{Q}_{1}^{\prime} x_{2}\right)\right)\right)\right)
$$

which is, $\mathrm{M} x_{1}, \mathrm{M} x_{2} \vdash \mathrm{M}\left\langle x_{1}, x_{2}\right\rangle$. Thus we have the theorem for $n=2$. We now assume the case of $n-1$, then $\mathrm{M} x_{1}, \ldots, \mathrm{M} x_{n-1} \vdash \mathrm{M}\left\langle x_{1}, \ldots, x_{n-1}\right\rangle$. Then by Axiom GA4,

$$
\mathbf{M} x_{1}, \ldots, \mathbf{M} x_{n} \vdash \mathbf{M}\left(\mathbf{Q}_{1}^{\prime}\left(\mathbf{U}\left(\mathbf{Q}_{1}^{\prime}\left\langle x_{1}, \ldots, x_{n-1}\right\rangle\right)\left(\mathbf{Q}_{1}^{\prime} x_{n}\right)\right)\right)
$$

and using the inductive hypothesis, $M x_{1}, \ldots, M x_{n} \vdash \mathbf{M}\left\langle x_{1}, \ldots, x_{n}\right\rangle$. Now we can state the theorem.

Theorem 6. If $\vdash \mathbf{M}_{n} \mathbf{X}$ then there exists a $\mathbf{Y}$ such that $\vdash \mathbf{M}_{1} \mathbf{Y}$ and $\mathbf{M} x_{1}, \ldots$, $\mathbf{M} x_{n} \vdash \mathbf{X} x_{1} \ldots x_{n} \sim \mathbf{Y}\left\langle x_{1}, \ldots, x_{n}\right\rangle$.

Proof. Let

$$
\mathbf{Y}=[t] \Sigma \mathbf{A}\left[x_{1}\right] \ldots \Sigma \mathbf{A}\left[x_{n}\right] . \mathbf{Q}_{1}^{\prime} t\left\langle x_{1}, \ldots, x_{n}\right\rangle \wedge \mathbf{X} x_{1} \ldots x_{n} .
$$

Now by Axioms $\mathrm{AQ} \prime$ and $\mathrm{AU}, \mathrm{A} t, \mathrm{~A} x_{1}, \ldots, \mathrm{A} x_{n} \vdash \mathrm{H}\left(\mathrm{Q}_{1}^{\prime} t\left\langle x_{1}, \ldots, x_{n}\right\rangle\right)$, and by Definition $\mathbf{M}_{n}, \mathbf{M}_{n} \mathbf{X}, \mathbf{A} x_{1}, \ldots, \mathbf{A} x_{n} \vdash \mathbf{H}\left(\mathbf{X} x_{1} \ldots x_{n}\right)$. Therefore,

$$
\mathbf{M}_{n} \mathbf{X}, \mathbf{A} t, \mathbf{A} x_{1}, \ldots, \mathbf{A} x_{n} \vdash \mathbf{H}\left(\mathbf{Q}_{1}^{\prime} t\left\langle x_{1}, \ldots, x_{n}\right\rangle \wedge \mathbf{X} x_{1} \ldots x_{n}\right),
$$

and so

$$
\mathbf{M}_{n} \mathbf{X}, \mathbf{A} t 1-\mathbf{H}\left(\Sigma \mathbf{A}\left[x_{1}\right] \ldots \Sigma \mathbf{A}\left[x_{n}\right] \mathbf{Q}_{1}^{\prime} t\left\langle x_{1}, \ldots, x_{n}\right\rangle \wedge \mathbf{X} x_{1} \ldots x_{n}\right),
$$

and by the deduction theorem, $M_{n} X \vdash F A H Y$. Now let

$$
\mathbf{Z}=\left[t, x_{1}, \ldots, x_{n}\right] \mathbf{Q}_{1}^{\prime} t\left\langle x_{1}, \ldots, x_{n}\right\rangle \wedge \mathbf{X} x_{1} \ldots x_{n},
$$

then, as $\mathbf{A} x_{1}, \ldots, \mathbf{A} x_{n}, \mathbf{M}_{n} \mathbf{X}, \mathbf{X} x_{1} \ldots x_{n} \vdash \mathbf{M} x_{1} \wedge \ldots \wedge \mathbf{M} x_{n}$, we have by the lemma and the axioms for equality, $\mathbf{A} t, \mathbf{A} x_{1}, \ldots, \mathbf{A} x_{n}, \mathbf{M}_{n} \mathbf{X}, \mathbf{Z} t x_{1} \ldots x_{n} \vdash \mathbf{M} t$. Then by (5) and the deduction theorem for $\mathbf{P}, \mathbf{M}_{n} \mathbf{X}, \mathbf{A} t, \mathbf{A} x_{1}, \ldots, \mathbf{A} x_{n} \vdash \mathbf{Z} t x_{1}$ $\ldots x_{n} \supset \mathbf{M} t$, and by $\vdash \mathrm{LA}$ and the deduction theorem for $\Xi, \mathrm{M}_{n} \mathbf{X}, \mathbf{A} t \vdash \Xi \mathbf{A}\left[x_{1}\right]$ $\ldots \Xi \mathbf{A}\left[x_{n}\right] . \mathbf{Z} t x_{1} \ldots x_{n} \supset \mathbf{M} t$. Then by (5), $\mathbf{A} t \vdash \mathbf{H}(\mathbf{M} t)$ and by Theorem 30 of $[2]$,

$$
\mathrm{M}_{n} \mathrm{X}, \mathrm{A} t \vdash \mathrm{Y} t \supset \mathrm{M} t .
$$

Therefore by (6) $\mathbf{M}_{n} \mathbf{X} \vdash \Xi A[t] . Y t \supset M t$. Thus by (6) again,

$$
M_{n} X \vdash M_{1} Y \text {. }
$$

Now $\mathbf{A} x_{1}, \ldots, \mathbf{A} x_{n}, \mathbf{Y}\left\langle x_{1}, \ldots, x_{n}\right\rangle \vdash \Sigma \mathbf{A}\left[u_{1}\right] \ldots \Sigma \mathbf{A}\left[u_{n}\right] . \quad \mathbf{Q}_{1}^{\prime}\left\langle x_{1}, \ldots, x_{n}\right\rangle$ 
$\left\langle u_{1}, \ldots, u_{n}\right\rangle_{\wedge} \mathbf{X} u_{1} \ldots u_{n}$. Also $\mathbf{A} x_{1}, \ldots, \mathbf{A} x_{n}, \mathbf{A} u_{1}, \ldots, \mathbf{A} u_{n}, \mathbf{Q}_{1}^{\prime}\left\langle x_{1}, \ldots\right.$, $\left.x_{n}\right\rangle\left\langle u_{1}, \ldots, u_{n}\right\rangle \vdash \mathbf{Q}_{1}^{\prime} x_{1} u_{1} \wedge \ldots \wedge \mathbf{Q}_{1}^{\prime} x_{n} u_{n}$, so since we have the correct grammatical conditions as in the proof of (7),

$$
\begin{gathered}
\mathbf{A} x_{1}, \ldots, \mathbf{A} x_{n}, \mathbf{A} u_{1}, \ldots, \mathbf{A} u_{n}, \mathbf{Q} \mathbf{Q}_{1}^{\prime}\left\langle x_{1}, \ldots, x_{n}\right\rangle\left\langle u_{1}, \ldots, u_{n}\right\rangle \wedge \\
\mathbf{X} u_{1} \ldots u_{n} \vdash \mathbf{X} x_{1} \ldots x_{n}
\end{gathered}
$$

and thus $\mathbf{A} x_{1}, \ldots, \mathbf{A} x_{n}, \mathbf{A} u_{1}, \ldots, \mathbf{A} u_{n} \vdash \mathbf{Q}_{1}^{\prime}\left\langle x_{1}, \ldots, x_{n}\right\rangle\left\langle u_{1}, \ldots, u_{n}\right\rangle \wedge \mathbf{X} u_{1}$ $\ldots u_{n} \supset \mathbf{X} x_{1} \ldots x_{n}$. Therefore by Theorem 26 of [2]

$$
\begin{gathered}
\mathbf{A} x_{1}, \ldots, \mathbf{A} x_{n} \vdash \Sigma \mathbf{A}\left[u_{1}\right] \ldots \Sigma \mathbf{A}\left[u_{n}\right] . \mathbf{Q}_{1}^{\prime}\left\langle x_{1}, \ldots, x_{n}\right\rangle\left\langle u_{1}, \ldots, u_{n}\right\rangle \wedge \\
\mathbf{X} u_{1} \ldots u_{n} . \supset \mathbf{X} x_{1} \ldots x_{n}
\end{gathered}
$$

and so

$$
\mathrm{M} x_{1}, \ldots, \mathrm{M} x_{n} \vdash \mathrm{Y}\left\langle x_{1}, \ldots, x_{n}\right\rangle \supset \mathrm{X} x_{1} \ldots x_{n} .
$$

Now $\mathrm{X} x_{1} \ldots x_{n}, \mathbf{M} x_{1}, \ldots, \mathbf{M} x_{n} \vdash \mathbf{Q}_{1}^{\prime}\left\langle x_{1}, \ldots, x_{n}\right\rangle\left\langle x_{1}, \ldots, x_{n}\right\rangle \wedge \mathbf{X} x_{1} \ldots x_{n}$, so $\mathbf{X} x_{1} \ldots x_{n}, \mathbf{M} x_{1}, \ldots, \mathbf{M} x_{n} \vdash \Sigma \mathbf{A}\left[u_{1}\right] \ldots \Sigma \mathbf{A}\left[u_{n}\right] . \mathbf{Q}_{1}^{\prime}\left\langle x_{1}, \ldots, x_{n}\right\rangle\left\langle u_{1}, \ldots\right.$, $\left.u_{n}\right\rangle \wedge \mathrm{X} x_{1} \ldots x_{n}$. Therefore $\mathrm{X} x_{1} \ldots x_{n}, \mathrm{M} x_{1}, \ldots, \mathrm{M} x_{n} \vdash \mathrm{Y}\left\langle x_{1}, \ldots, x_{n}\right\rangle$, and by $\mathrm{M}_{n} \mathrm{X}, \mathrm{M} x_{1}, \ldots, \mathrm{M} x_{n} \vdash \mathrm{X} x_{1} \ldots x_{n} \supset \mathrm{Y}\left\langle x_{1}, \ldots, x_{n}\right\rangle$. Thus by (9),

$$
\mathbf{M} x_{1}, \ldots, \mathbf{M} x_{n} \vdash \mathbf{X} x_{1} \ldots x_{n} \sim \mathbf{Y}\left\langle x_{1}, \ldots, x_{n}\right\rangle \text {. }
$$

Now we combine Theorems 5 and 6 to give us:

Theorem 7. If $\phi\left(x_{1}, \ldots, x_{n}\right)$ is a ppf containing no free variables other than at most $x_{1}, \ldots, x_{n}$ then there exists a class $\mathbf{Y}$ such that $\mathbf{M} x_{1}, \ldots$, $\mathrm{M} x_{n} \vdash \mathrm{Y}\left\langle x_{1}, \ldots, x_{n}\right\rangle \sim \phi\left(x_{1}, \ldots, x_{n}\right)$.

This is the general existence theorem exactly as Gödel has it. If we apply the deduction theorem for $P$ to the formula in Theorem 7 several times we get:

$$
\vdash \Xi \mathbf{A}\left[x_{1}\right] \ldots \Xi \mathbf{A}\left[x_{n}\right] . \mathbf{M} x_{1} \supset \ldots \mathbf{M} x_{n} \supset . \mathbf{Y}\left\langle x_{1}, \ldots, x_{n}\right\rangle \sim \phi\left(x_{1}, \ldots, x_{n}\right) .
$$

This theorem from which we derive all the axioms of the B group is also in the correct translated form. We therefore have proved the general theorem for the Gödel system.

Theorem 8. If $T$ is a theorem of the Gödel system of set theory and $N$ is the set of grammatical conditions for $T^{\prime}$, the translation of $T$ into the combinatory system, then $N \vdash T^{\prime}$.

\section{REFERENCES}

[1] Bernays, P., Axiomatic Set Theory, Amsterdam (1958).

[2] Bunder, M. W., "Propositional and predicate calculuses based on combinatory logic ,' Notre Dame Journal of Formal Logic, vol. XV (1974), pp. 25-34.

[3] Bunder, M. W., "A deduction theorem for restricted generality," Notre Dame Journal of Formal Logic, vol. XIV (1973), pp. 341-346. 
[4] Bunder, M. W., "A paradox in illative combinatory logic," Notre Dame Journal of Formal Logic, vol. XI (1970), pp. 467-470.

[5] Cogan, E. J., "A formalization of the theory of sets from the point of view of combinatory logic," Zeitschrift für Mathematische Logik und Grundlagen der Mathematik, vol. 1 (1955), pp. 198-240.

[6] Curry, H. B., and R. Feys, Combinatory Logic, Amsterdam (1958).

[7] Fraenkel, A., and Y. Bar Hillel, Foundations of Set Theory, Amsterdam (1958).

[8] Gödel, K., The Consistency of the Continuum Hypothesis, Princeton (1940).

[9] Titgemeyer, R., "Uber einen Widerspruch in Cogan's Darstellung der Mengenlehre," Zeitschrift für Mathematische Logik und Grundlagen der Mathematik, vol. 7 (1961), pp. 161- 163.

[10] Zermelo, E., "Untersuchungen Uber die Grundlagen der Mengelehre," Mathematische Annalen, vol. 65 (1908), pp. 261-281.

Wollongong University College

Wollongong, New South Wales, Australia 